production. ${ }^{2}$ Oxygen treatment undoubtedly relieves the symptoms and the cysts regress. ${ }^{1}$ Since hydrogen is more diffusible than oxygen the mechanism is difficult to explain unless oxygen prevents the production of hydrogen by impairing a facultative anaerobic organism. The condition regresses after (unrelated) treatment with ampicillin, ${ }^{3}$ and animal models can be made to simulate the condition using Clostridium perfringens. ${ }^{4}$ That the condition remains uncommon in patients with severe respiratory disease suggests that hydrogen "retention" is not a major factor. Local abnormalities are probably responsible and a genetic link has been suggested. ${ }^{5}$ An underlying structural difference in the collagen of the supporting tissue of the gut and lung may be responsible.

The dramatic response of these two patients to metronidazole is further evidence to support the thesis that the cysts of pneumatosis coli are maintained by gas produced by anaerobic organisms. Since the condition relapses after treatment with oxygen ${ }^{2}$ it is likely to relapse also after that with metronidazole, because both are probably effective for the same reason. But metronidazole provides a simple and safe treatment without recourse to hospital admission for oxygen treatment.

I thank Mr A G Cox for help in preparing this paper and for permission to report on patients under his care.

Footnote Since writing this report we have treated a third patient with intermittent metronidazole with pronounced symptomatic improvement.

${ }^{1}$ Wyatt AP. Prolonged symptomatic and radiological remission of colonic gas cysts after oxygen therapy. Brf Surg 1975;62:837-9.

2 Van Der Linden W, Hoflin F. Pneumatosis cystoides coli recurs after oxygen treatment. A clue to pathogenesis? Eur Surg Res 1978;10: 225-9.

${ }^{3}$ Holt S, Stewart IC, Heading RC, Macpherson AIS. Resolution of primary pneumatosis coli. $\mathcal{F} R$ Coll Surg Edinb 1978;23:297-9.

4 Yale CE, Balish E, Wu JP. The bacterial etiology of pneumatosis cystoides intestinalis. Arch Surg 1974;109:89-94.

${ }^{5}$ Underwood JW, Finnis F, Scott W. Pneumatosis coli: a familial association. Brf Surg 1978;65:64-5.

(Accepted 15 fanuary 1980)

Northwick Park Hospital and Clinical Research Centre, Harrow, Middlesex HA1 3UJ

B W ELLIS, MB, FRCS, senior registrar in surgery

\section{Endoscopic retrograde cholangiopancreatography for unexplained upper abdominal pain}

The management of patients with abdominal pain of unknown aetiology is difficult and often a series of expensive investigations is carried out. Endoscopic retrograde cholangiopancreatography (ERCP) is often suggested to exclude pancreatic disease and gall stones not seen on cholecystography. ${ }^{1}$ In our experience, however, this investigation is usually unhelpful, and we have therefore reviewed our results.

\section{Patients, methods, and results}

Out of 806 ERCP examinations attempted during the past five years 140 had been requested for patients who had undiagnosed upper abdominal pain. Seventy of the 140 had not undergone previous biliary surgery (group 1). Their liver function tests; serum amylase concentration; and the results of cholecystogram, barium meal, or upper gastrointestinal endoscopy examinations were normal. The biliary or pancreatic duct systems, or both, were opacified in $59(84 \%)$ of them. The diagnoses are shown in the table. In seven (10\%) a diagnosis was made at ERCP: three had peptic ulceration and four had pancreatic disease (benign stricture, carcinoma, chronic pancreatitis, abnormal medial wall of the duodenum). Out of the 18 in whom some psychiatric disorder was diagnosed 12 improved after treatment with psychotropic drugs. Three of the remainder had a personality disorder and three were depressed but failed to attend for follow-up. In two patients in whom ERCP was unsuccessful pancreatic disease was diagnosed later by other methods (both had chronic pancreatitis diagnosed at laparotomy or by ultrasonography). The remaining 70 of the
140 patients with undiagnosed abdominal pain had previously undergone biliary surgery. In 45 of them some abnormality of liver function tests had suggested the need for ERCP. But in 25 liver tests were normal (group 2). Their final diagnoses were similar to those in the patients in group 1 (table).

Diagnoses and outcome in 70 patients examined by ERCP who had not undergone biliary surgery (group 1) and 25 patients who had had previous surgery (group 2)

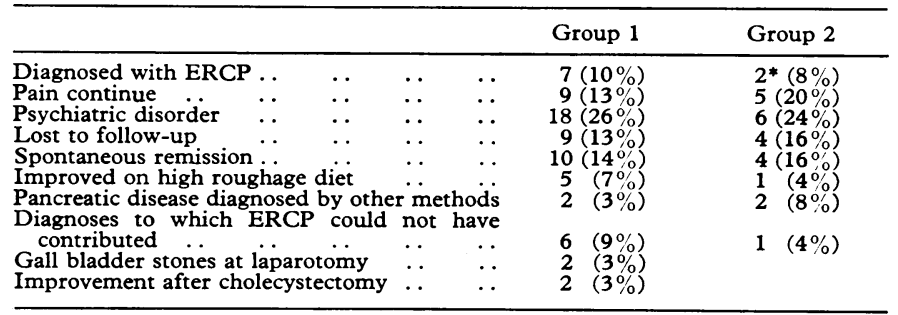

*Common bile duct stones; duodenitis.

In both groups one or more ducts were cannulated in $84 \%$ of cases. There was $79 \%$ success in opacifying the pancreatic duct, similar to the $78 \%-98 \%$ of other series, ${ }^{2}$ even though the examinations were made by different operators of varying experience. We confirmed observations that the biliary tree is more easily opacified after biliary surgery ( $44 \%$ group 1 , $60 \%$ group 2 ). When the results in the patients in whom both ducts were opacified are compared with those in whom only one or neither duct was opacified the proportions of the groups remain similar. Thus it is unlikely that we have underdiagnosed pancreatic disease because of technical failure.

\section{Comment}

The cause of abdominal pain is often never found. The pain may continue even though every routine test result is normal. After expensive investigations ERCP is considered to try to exclude gall stones or pancreatic disease. Our results show that the value of ERCP in making a definite diagnosis is low, although a normal ERCP result may strengthen the doctor's determination to treat with psychotropic drugs or a high-fibre diet. Indeed, our three patients with peptic ulcer could have been diagnosed by routine upper gastrointestinal endoscopy, while the value of diagnosing pancreatic carcinoma early is doubtful. ${ }^{3}$ Nevertheless, laparotomy is unlikely to give better results than ERCP, ${ }^{4}$ and has a greater morbidity and mortality. Careful prior selection of patients by ultrasound or computed tomography might increase the value of ERCP, ${ }^{5}$ but neither is cheap and the availability of computed tomography is restricted. ERCP is more likely to be of value after biliary tract surgery since the results of intravenous cholangiography are often uncertain. But even in these patients we found few abnormalities among those with normal liver function tests.

We suggest that before submitting patients with undiagnosed abdominal pain to ERCP the exasperated doctor should try treatment with bran or antidepressant drugs.

This work was presented to the British Society of Gastroenterology in March 1979. We thank Drs P B Cotton, A T R Axon, M D Hellier, and I T Gilmore, who performed some of the examinations, and ICI Ltd for financial support. P W N Keeling is a Medical Research Council training fellow.

${ }^{1}$ Smith R, Williams R, Cotton PB. Gallstone pancreatitis with normal biliary radiology. Br $\mathcal{F}$ Surg 1976;63:861-3.

${ }^{2}$ Kasugai T. Recent advances in the endoscopic retrograde cholangiopancreatography. Digestion 1975;13:76-99.

${ }^{3}$ Salmon PR. Re-evaluation of endoscopic retrograde cholangiopancreatography as a diagnostic method. In: Schiller KFR, ed. Clinics in gastroenterology. No 7, vol 3. Philadelphia: WB Saunders, 1978:651-66.

${ }^{4}$ Anonymous. Diagnostic laparotomy. Br Med $\mathcal{F} 1977$;ii:144-5.

5 Levitt RG, Geisse GG, Sagell SS, et al. Complementary use of ultrasound and computed tomography in studies of the pancreas and kidney. Radiology 1978;126:149-52.

(Accepted 10 January 1980)

Gastrointestinal Research Unit, Rayne Institute, St Thomas's

Hospital, London SE1 7EH

JOHN BULL, MB, MRCP, senior medical registrar

P W N KEELING, MRCP, MRCPI, honorary senior registrar

R P H THOMPSON, DM, FRCP, consultant physician 